\title{
Adaptation to sensory input tunes visual cortex to criticality
}

\author{
Woodrow L. Shew ${ }^{1 \star}$, Wesley P. Clawson ${ }^{1}$, Jeff Pobst ${ }^{2}$, Yahya Karimipanah ${ }^{2}$, Nathaniel C. Wright ${ }^{2}$ \\ and Ralf Wessel ${ }^{2}$
}

\begin{abstract}
A long-standing hypothesis at the interface of physics and neuroscience is that neural networks self-organize to the critical point of a phase transition, thereby optimizing aspects of sensory information processing ${ }^{1-3}$. This idea is partially supported by strong evidence for critical dynamics observed in the cerebral cortex ${ }^{4-10}$, but the impact of sensory input on these dynamics is largely unknown. Thus, the foundations of this hypothesis-the self-organization process and how it manifests during strong sensory input-remain unstudied experimentally. Here we show in visual cortex and in a computational model that strong sensory input initially elicits cortical network dynamics that are not critical, but adaptive changes in the network rapidly tune the system to criticality. This conclusion is based on observations of multifaceted scaling laws predicted to occur at criticality ${ }^{4,11}$. Our findings establish sensory adaptation as a self-organizing mechanism that maintains criticality in visual cortex during sensory information processing.
\end{abstract}

Sensory nervous systems adapt, dynamically tuning interactions among large networks of neurons, to cope with a changing environment ${ }^{12,13}$. The principles governing such adaptation at the macroscopic level of neuronal network dynamics are not well understood. Computational models and theory suggest that such adaptation can maintain critical network dynamics ${ }^{14-16}$, but these previous studies did not consider the strongly driven regime that is expected during intense sensory input. Indeed, sufficiently strong input may increase the overall excitability of a network by bringing neurons closer to their firing thresholds and potentially tipping the network into a high firing rate regime that is inconsistent with critical dynamics (Supplementary Information 1). Thus, the question remains: does strong sensory input drive cortical network dynamics away from criticality or can adaptation counteract this tendency and maintain the critical regime?

Here we addressed this question in turtle visual cortex and in a companion computational model. In our experiments, we obtained long-duration recordings of population neural activity (local field potential, LFP) using a microelectrode array inserted into the geniculo-recipient dorsal cortex (visual cortex) of the turtle eyeattached whole-brain ex vivo preparation ${ }^{17}$ (Fig. 1a and Supplementary Information 2). We measured multi-scale spatiotemporal patterns of neural activity while visually stimulating the retina. Similarly, in our model we studied changes in neural network activity in response to changes in external input. Experimentally, and in the model, we assessed whether the measured dynamics were near or far from criticality. For this, we examined statistics and spatiotemporal scaling laws of 'neuronal avalanches', which are bouts of elevated population activity with correlations in space and time ${ }^{5}$ (Fig. 1b).
In brief, a neuronal avalanche is defined as a group of LFP peaks, occurring on any electrode, irrespective of location, and separated by inter-peak intervals less than a specified time (Methods). For experiments in which spikes (that is, multiunit activity) were also measurable, we confirmed that the rate of LFP peaks increases with the rate of spikes (Supplementary Information 3). Thus, a period of time with many LFP peaks-for example, a neuronal avalanchereflects an increase in population spike rate in the cortex.

At the onset of stimulation, we observed that LFP amplitude, LFP peak rate, and avalanches were typically large scale-not consistent with critical dynamics - during a transient period (Fig. 1c-e and Supplementary Information 4). More specifically, avalanche sizes $S$ and durations $D$ were often bimodally distributed during the transient (Fig. 1f,g and Supplementary Information 5). Following this large-scale transient response, LFP amplitude decreased and avalanches became more diverse in spatiotemporal scale (Fig. 1e), resulting in power-law distributions, $P(S) \sim S^{-\tau}$ (Fig. 1f) and $P(D) \sim D^{-\alpha}$ (Fig. 1g) over a wide range of sizes and durations. This fact is supported by rigorous maximum likelihood fitting methods ${ }^{10,18}$ and strict statistical criteria for fit quality ( $q>0.1$, Methods).

These conclusions held for nine turtles and four types of visual stimuli ( $n=13$ data sets; complex movies, static grey screen, diffuse flashes, moving dots) with power-law quality values $q=0.31 \pm 0.13$ (mean \pm s.d.). Importantly, the different visual stimuli had very different spatiotemporal structure, yet all resulted in power-law avalanche distributions. This indicates that the power laws were due to inherent neuronal network dynamics rather than externally imposed statistics of the stimulus. Notably, randomizing the recorded LFP peak times abolished the power-law distributions of avalanche size and duration, thus demonstrating the importance of correlations, (Fig. 1f,g). Moreover, activity recorded outside visual cortex was not power-law distributed (Supplementary Information 6).

What biophysical mechanisms could mediate self-organization towards scale-free population activity during visual processing? To address this question, we investigated a parsimonious model network of probabilistic integrate-and-fire neurons with all-to-all connectivity $^{6,19,20}$ (Fig. 2a). A subset of neurons (20\%) was inhibitory. Motivated by previous experiments ${ }^{21}$ and models ${ }^{14}$, we modelled adaptation as short-term synaptic depression with recovery (Methods). However, our model differed from previously studied models, as detailed in Supplementary Information 7. We studied how the model dynamics and avalanche statistics change as a result of increasing input rate. During a transient period after increasing the input rate, the population spike rate increased and synapses depressed (Fig. 2b,c). During the transient, avalanches also increased markedly in size and duration (Fig. 2d), qualitatively similar to the experimental observations (Fig. 1e). 
a

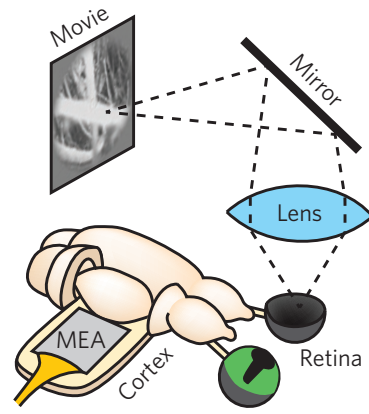

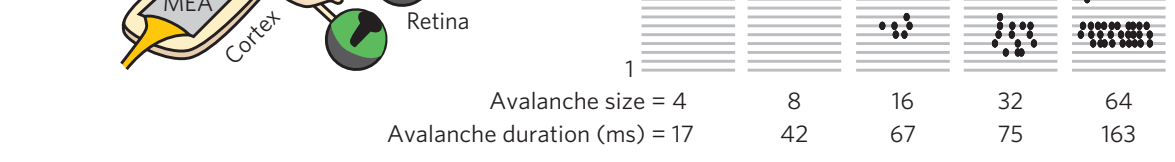

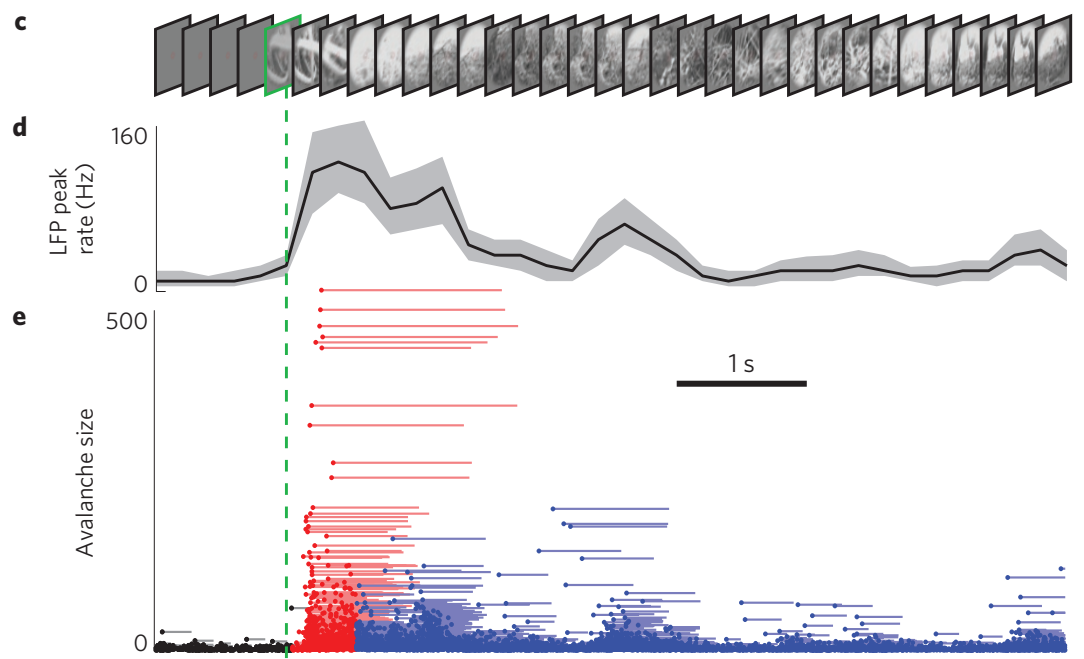

$\mathbf{f}$
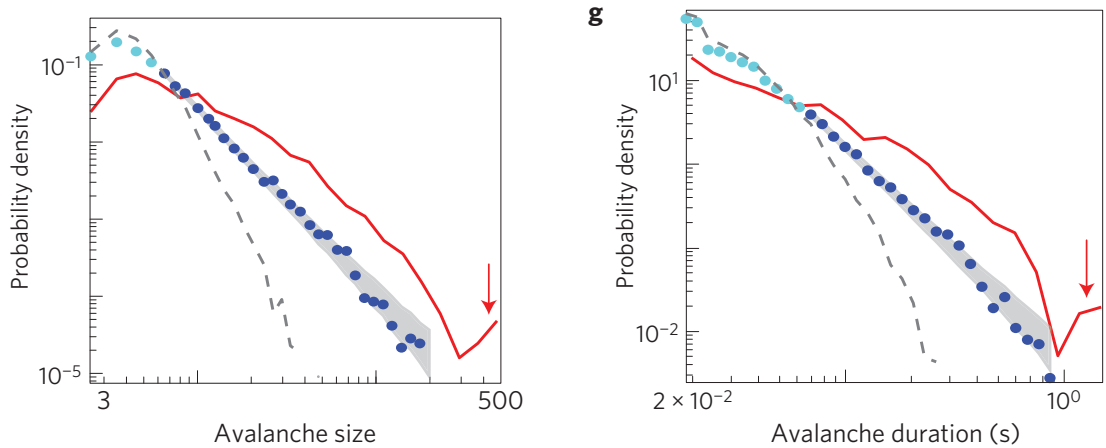

Figure 1 | Visually driven network dynamics are power-law distributed after non-power-law transient. a, Ex vivo whole brain with eyes attached. Visual stimuli are projected onto the intact retina while activity is recorded with a 96-channel microelectrode array (MEA) inserted into the unfolded visual cortex. b. Avalanches are defined as spatiotemporal clusters of large amplitude LFP peaks (black dots). Five example avalanches are shown, with one dot per LFP peak. Avalanche size = number of LFP peaks; duration = time between the first and last peaks. SD, standard deviation. $\mathbf{c}$, Natural movie visual stimulus (subset of 1 frame per 200 ms shown). d, At the start of the movie (green dashed line), there is a transient increase in stimulus-triggered average LFP peak rate. The average is over 315 movie presentations. The grey region delineates quartiles. e, Stimulus-triggered avalanche size time series reveals the tendency for very large avalanches during the transient response (red). Later, during the visually driven steady state, less extreme avalanches occur (blue). Each point represents one avalanche. The line following each point indicates avalanche duration. Responses to 315 repeats of movie stimulation are overlaid. $\mathbf{f}, \mathbf{g}$, Probability density functions for sizes and durations of avalanches during the transient response (red line) and during the visually driven steady state (light and dark blue dots). Large avalanches (arrow) occur during the transient response, yielding bimodal distributions of avalanche sizes and durations. Avalanches during the visually driven steady state are power-law distributed over the range indicated with dark blue dots. Grey shading indicates the range (0.05-0.95) of expected probabilities for a perfect power law with the same number of samples as the experiment. Jittering the times of LFP peaks destroys the power law (dashed line) by abolishing large-sized avalanches. Panels $\mathbf{d}-\mathbf{g}$ are from one experiment with one turtle.

Avalanche size and duration distributions during the transient period exhibited a distinct bimodal character consisting of small and large avalanches (Fig. 2e,f), qualitatively similar to what we found experimentally (Fig. 1f,g) and inconsistent with a power law. Following the transient jump in population activity, the network dynamically reached a new steady state of population activity and average synaptic strength (Fig. 2b,c). In this driven steady state, during continued high external input rate, the simulated neuronal avalanche size and duration distributions were power laws $(q>0.1)$ (Fig. 2e,f and Supplementary Information 7). We note that the 
a

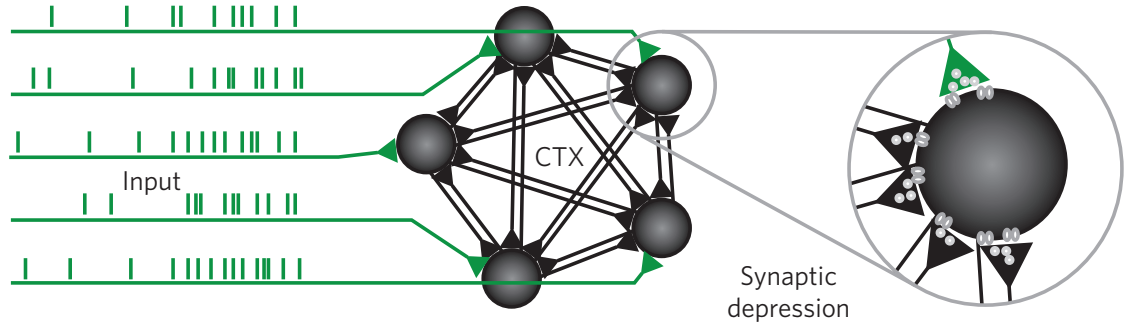

b
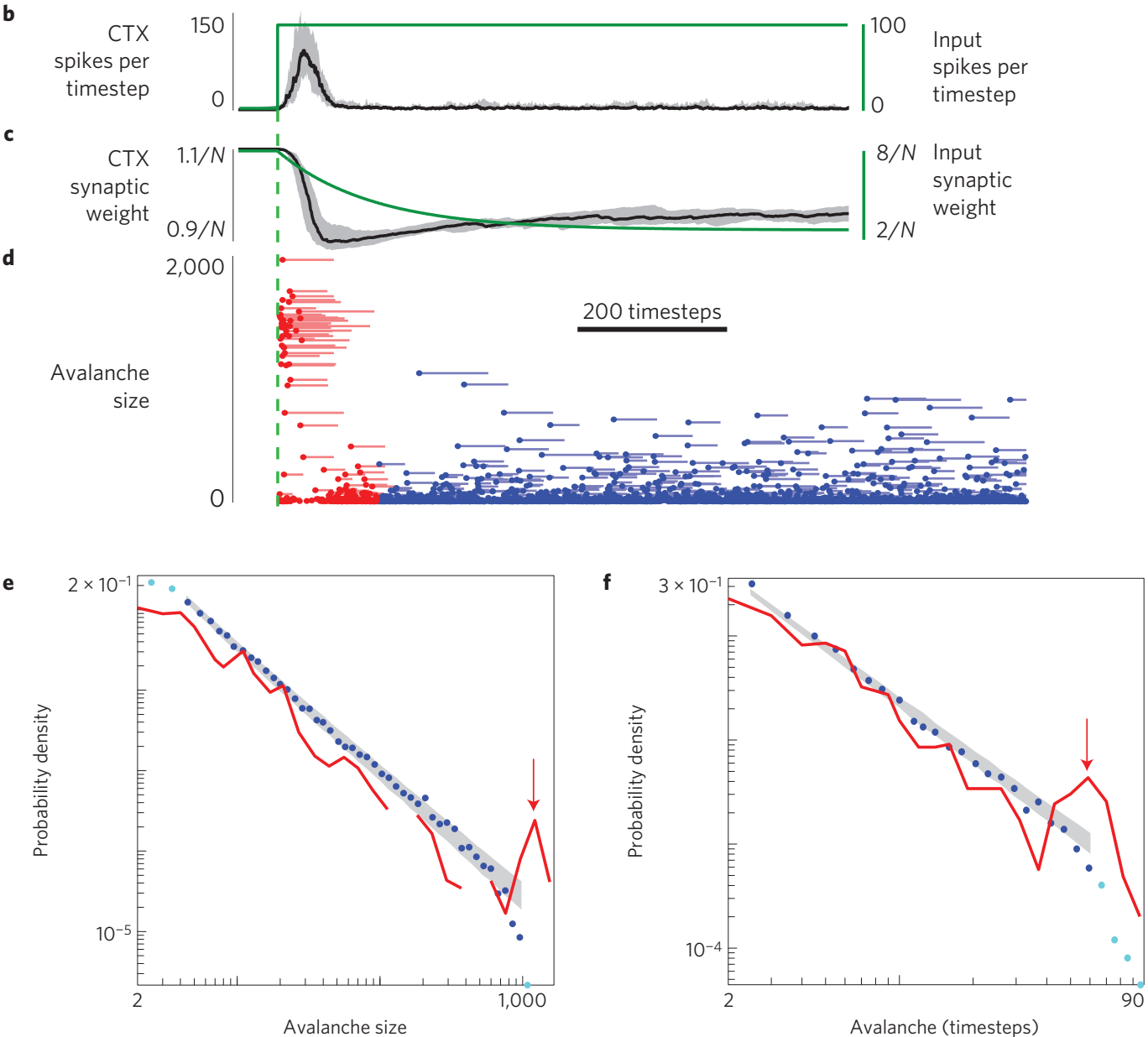

Figure 2 | Depressing synapses tune model dynamics to critical regime after non-critical transient. a, Conceptual cartoon illustrating model features, including recurrent synapses (black) and input synapses (green) that change strength according short-term depression. b, A step increase in input (green, input spikes per timestep for the whole network) causes a transient increase in the population spike rate of the network (black, median). The time series is averaged over 40 trials. The grey region delineates quartiles. c, Following the input rate increase, there is a relatively slow decrease in median synaptic strength for both recurrent (black) and input (green) synapses (average over 40 trials). The grey region delineates quartiles. The dashed line marks stimulus onset. $N=1,000$ is the number of neurons in the model. $\mathbf{d}$, Stimulus-triggered avalanche time series. During a transient period after an increase in input rate, avalanches of very large size occur (red). e, Distributions of avalanche size during the transient period (red) reveal a 'bump' in the avalanche size distribution at large size (arrow). Avalanche sizes are power-law distributed after synapses have adapted (dark blue points are within power-law fit range, light blue are outside the fit range). Grey shading indicates the range (0.05-0.95) of expected probabilities for a perfect power law with the same number of samples. f, Avalanche durations follow a similar trend. Model parameters: low input $R=0.05$; high input $R=100$; default synapses $\Lambda_{0}=1.1 ; 30 \%$ subsampling; 5,000 timesteps computed after increase in $R$.

similarity of model and experimental results persists even when the model is subsampled-that is, many of the spikes are deleted before analysing avalanches, such that the rate of model spikes matches the experimentally observed LFP peak rate (Supplementary Information 7).

In the model, a sufficiently strong increase in input rate transiently tips the system into a regime without critical dynamics (Supplementary Information 1). Adaptation then tunes the system to a critical regime. Given the similarity between our model results and our experiment, it is tempting to conclude that the experimentally observed power-law avalanche distributions occurred because adaptation tunes the visual cortex to criticality. However, caution is called for, because power laws provide necessary, but insufficient evidence for the critical regime ${ }^{22-24}$. Therefore, additional tests are needed to determine whether criticality underlies the experimentally observed power laws. 
Experiment
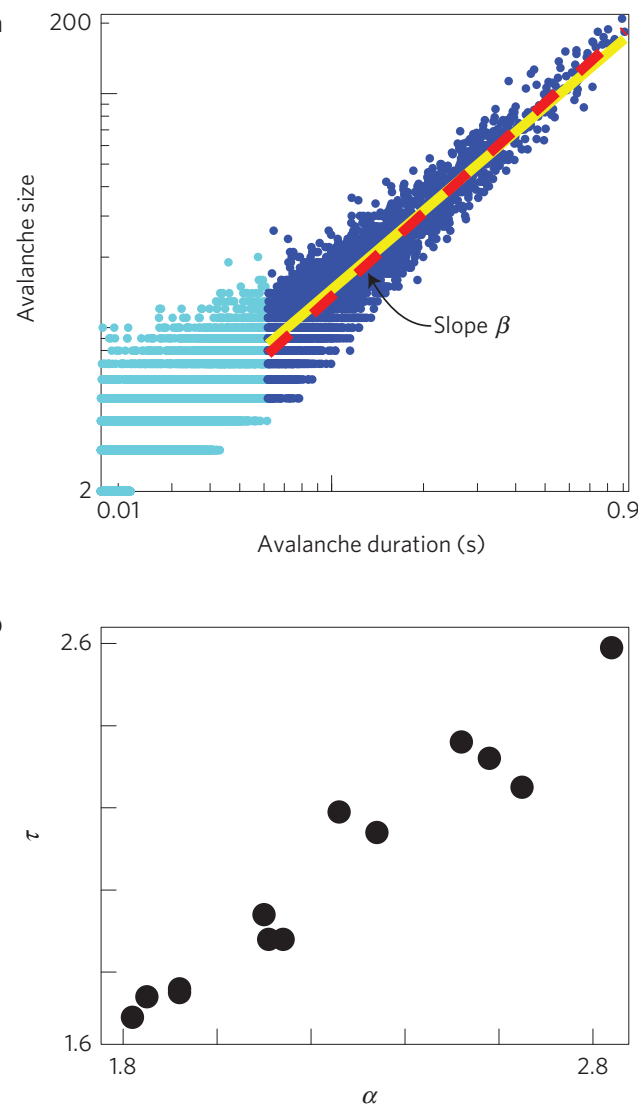

c

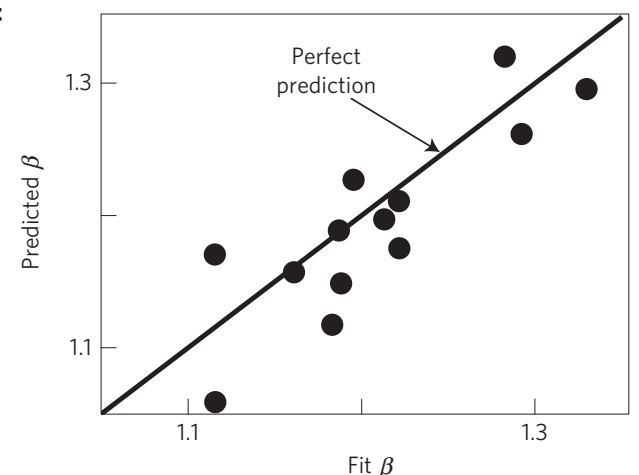

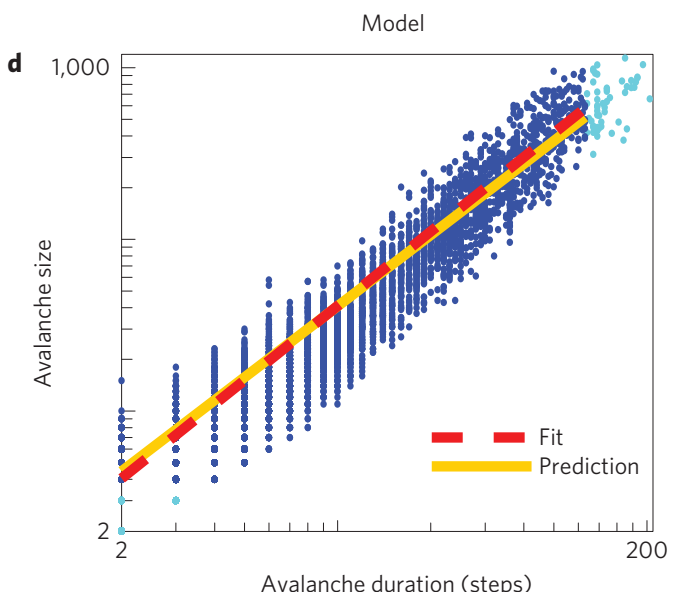

e

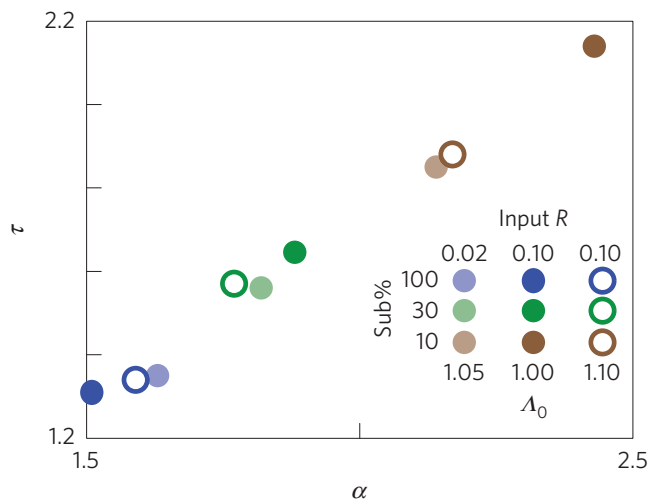

$\mathbf{f}$

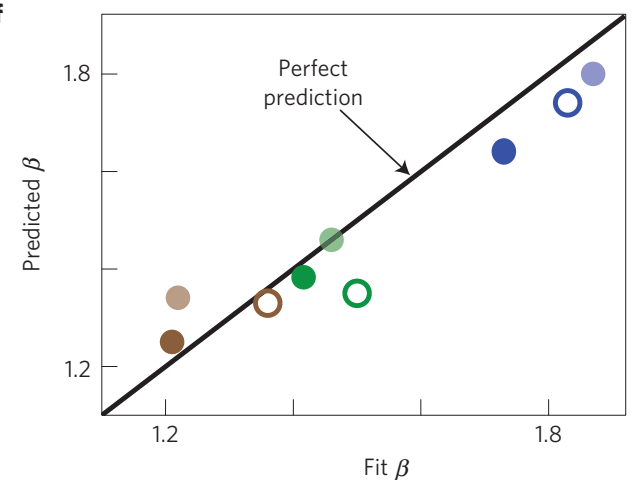

Figure 3 | Steady state visually driven avalanches follow predictions for critical regime. a, Each point indicates the size and duration of one avalanche (from one experiment, one turtle, as in Fig. 1). Avalanches from the visually driven steady state are shown. The linear relationship on logarithmic axes

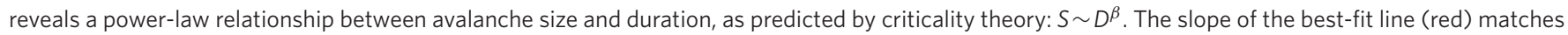
with the predicted $\beta=(\alpha-1) /(\tau-1)$ (yellow). b, In different experiments, different values were found for duration exponents $\alpha$ and size exponents $\tau$. c, Different best-fit $\beta$ were found for different turtles. Predicted $\beta$ matched the best-fit $\beta$ in the visually driven steady state. The line indicates an identical match. $\mathbf{d}-\mathbf{f}$, Computational model exhibits the same relationship among power-law exponents as found experimentally. Different size and duration exponents (e) were obtained by varying the input rate $R$, the degree of subsampling, and default synapse strengths $\Lambda_{0}$ (see inset legend).

Two such tests arise from a particular relationship between the size and duration of avalanches, which is predicted to occur at criticality,11 and confirmed by our model (Fig. 3d-f and Supplementary Information 1 and 7). First, the average avalanche size increases with duration according to a specific function $S \sim D^{\beta}$. Second, the exponent $\beta$ is predicted to depend on the exponents $\tau$ and $\alpha$ as $\beta=(\alpha-1) /(\tau-1)$.

Our experiments confirmed both these predictions (Fig. 3a-c). In addition, we confirmed a third prediction based on empirically estimated branching parameters ${ }^{25}$ in Supplementary Information 8. First, we showed that avalanche size scales with duration according to a power law (Fig. 3a). Second, we determined the 'best-fit $\beta$ ' from the size versus duration data for each experiment (Fig. 3a). Next we computed the 'predicted $\beta$ ' using the observed exponents $\tau$ and $\alpha$. For different turtles and different visual stimuli, we obtained a range of exponents; $1.7<\tau<2.6$ and $1.8<\alpha<2.8$ (Fig. 3b). Importantly, the observed values of $\tau$ and $\alpha$ provided a good prediction, $\beta=(\alpha-1) /(\tau-1)$, of the best-fit $\beta$ for all experiments (Fig. $3 \mathrm{c}$ and Supplementary Information 5).

The experimentally observed range of the values for the exponents $\tau$ and $\alpha$ (Fig. 3b) raises an important question as to the origin of this variability. In the model, we demonstrated that $\tau$ and 
$\alpha$ depended on three factors (Fig. 3e): the subsample fraction, the external input rate, and the default synaptic strength (parameterized by the largest eigenvalue $\Lambda_{0}$ of the default synaptic weight matrix ${ }^{26}$, see Supplementary Information 7). Subsampling — that is, creating avalanche distributions based on spikes from a subset of all model neurons-tended to increase $\tau$ and $\alpha$, consistent with previous studies $^{25,27}$. The effects of input rate and default synapse strengths depended on the level of subsampling. Importantly, the various combinations of $\tau$ and $\alpha$ observed in the model preserve the size versus duration scaling relationship (Fig. 3f), similar to what we found experimentally (Fig. 3c). We note that the near-linear relationship between $\tau$ and $\alpha$ (Fig. 3b,e) has not, to our knowledge, been predicted theoretically.

The close match between our experimental observations and our model results suggests that adaptation plays a crucial role in tuning cortical circuits towards the critical regime during vision. Why should adaptation in sensory cortex tune the network to operate near a critical regime? Previous computational studies and cortex slice experiments suggest that the critical regime optimizes several aspects of information processing (reviewed in ref. 3), including dynamic range $e^{20,26,28}$ and information transmission ${ }^{5,6}$. Our observation that transient response to stimulus onset is not critical suggests that sensory cortex dynamically adapts to gain the functional benefits of critical dynamics during strong sensory input.

The critical regime has long been hypothesized to be a target of homeostatic processes in neural networks. This could be achieved by some 'top-down' mechanism (for example, neuromodulators such as dopamine $\mathrm{e}^{29}$ ) that tunes the network or as the result of local self-organization ${ }^{14,16,30,31}$. In either case, one concern with this hypothesis has been that, theoretically, the critical regime occupies an infinitesimal volume in state space (the boundary between two different regimes), which may be too small a target to hit for a real biological tuning process contending with noise and imperfections. Recent theoretical findings mitigate this concern, showing that in networks with complex structure, the critical regime expands, occupying a substantial region (Griffiths phase) in state space ${ }^{32}$. Thus, our experiments, together with previous theory, establish the critical regime as a viable target for adaptive self-tuning during cortical sensory information processing.

\section{Methods}

Methods and any associated references are available in the online version of the paper.

Received 20 August 2014; accepted 19 May 2015; published online 22 June 2015

\section{References}

1. Plenz, D., Niebur, E. \& Schuster, H. G. Criticality in Neural Systems (Wiley, 2014).

2. Beggs, J. M. The criticality hypothesis: How local cortical networks might optimize information processing. Phil. Trans. R. Soc. A 366, 329-343 (2008).

3. Shew, W. L. \& Plenz, D. The functional benefits of criticality in the cortex. Neuroscientist 19, 88-100 (2013).

4. Friedman, N. et al. Universal critical dynamics in high resolution neuronal avalanche data. Phys. Rev. Lett. 108, 1-5 (2012).

5. Beggs, J. M. \& Plenz, D. Neuronal avalanches in neocortical circuits. J. Neurosci. 23, 11167-11177 (2003).

6. Shew, W. L., Yang, H., Yu, S., Roy, R. \& Plenz, D. Information capacity and transmission are maximized in balanced cortical networks with neuronal avalanches. J. Neurosci. 31, 55-63 (2011).

7. Petermann, T. et al. Spontaneous cortical activity in awake monkeys composed of neuronal avalanches. Proc. Natl Acad. Sci. USA 106, 15921-15926 (2009).

8. Haimovici, A., Tagliazucchi, E., Balenzuela, P. \& Chialvo, D. R. Brain organization into resting state networks emerges at criticality on a model of the human connectome. Phys. Rev. Lett. 110, 178101 (2013).
9. Priesemann, V., Valderrama, M., Wibral, M. \& Le Van Quyen, M. Neuronal avalanches differ from wakefulness to deep sleep - evidence from intracranial depth recordings in humans. PLoS Comput. Biol. 9, e1002985 (2013).

10. Klaus, A., Yu, S. \& Plenz, D. Statistical analyses support power law distributions found in neuronal avalanches. PLoS ONE 6, e19779 (2011).

11. Sethna, J. P., Dahmen, K. A. \& Myers, C. R. Crackling noise. Nature 410, 242-250 (2001)

12. Kohn, A. Visual adaptation: Physiology, mechanisms, and functional benefits. J. Neurophysiol. 97, 3155-3164 (2007).

13. Bialek, W. Biophysics: Searching for Principles (Princeton Univ. Press, 2012).

14. Levina, A., Herrmann, J. M. \& Geisel, T. Dynamical synapses causing self-organized criticality in neural networks. Nature Phys. 3, 857-860 (2007).

15. Levina, A., Herrmann, J. \& Geisel, T. Phase transitions towards criticality in a neural system with adaptive interactions. Phys. Rev. Lett. 102, 118110 (2009).

16. Meisel, C. \& Gross, T. Adaptive self-organization in a realistic neural network model. Phys. Rev. E 80, 1-6 (2009).

17. Saha, D., Morton, D., Ariel, M. \& Wessel, R. Response properties of visual neurons in the turtle nucleus isthmi. J. Comp. Physiol. A 197, 153-165 (2011).

18. Clauset, A., Shalizi, C. R. \& Newman, M. E. J. Power-law distributions in empirical data. SIAM Rev. 51, 661-703 (2009).

19. Haldeman, C. \& Beggs, J. M. Critical branching captures activity in living neural networks and maximizes the number of metastable states. Phys. Rev. Lett. 94, 058101 (2005).

20. Kinouchi, O. \& Copelli, M. Optimal dynamical range of excitable networks at criticality. Nature Phys. 2, 348-351 (2006).

21. Chung, S., Li, X. \& Nelson, S. B. Short-term depression at thalamocortical synapses contributes to rapid adaptation of cortical sensory responses in vivo. Neuron 34, 437-446 (2002).

22. Sornette, D. Critical Phenomena in Natural Sciences: Chaos, Fractals, Selforganization and Disorder: Concepts and Tools (Springer, 2006).

23. Stumpf, M. P. H. \& Porter, M. A. Mathematics. Critical truths about power laws. Science 335, 665-666 (2012).

24. Beggs, J. M. \& Timme, N. Being critical of criticality in the brain. Front. Physiol. 3, 163 (2012).

25. Priesemann, V. et al. Spike avalanches in vivo suggest a driven, slightly subcritical brain state. Front. Syst. Neurosci. 8, 108 (2014).

26. Larremore, D. B., Shew, W. L. \& Restrepo, J. G. Predicting criticality and dynamic range in complex networks: Effects of topology. Phys. Rev. Lett. 106, 1-4 (2011).

27. Ribeiro, T. L., Ribeiro, S., Belchior, H., Caixeta, F. \& Copelli, M. Undersampled critical branching processes on small-world and random networks fail to reproduce the statistics of spike avalanches. PLoS ONE 9, e94992 (2014).

28. Shew, W. L., Yang, H., Petermann, T., Roy, R. \& Plenz, D. Neuronal avalanches imply maximum dynamic range in cortical networks at criticality. J. Neurosci. 29, 15595-15600 (2009).

29. Gireesh, E. \& Plenz, D. Neuronal avalanches organize as nested theta- and beta/gamma-oscillations during development of cortical layer 2/3. Proc. Natl Acad. Sci. USA 105, 7576-7581 (2008).

30. Bak, P., Tang, C. \& Wiesenfeld, K. Self-organized criticality: An explanation of the 1/f noise. Phys. Rev. Lett. 59, 381-384 (1987).

31. Bornholdt, S. \& Röhl, T. Self-organized critical neural networks. Phys. Rev. E 67, 1-5 (2003)

32. Moretti, P. \& Muñoz, M. A. Griffiths phases and the stretching of criticality in brain networks. Nature Commun. 4, 2521 (2013).

\section{Acknowledgements}

We thank J. Clark for comments on previous versions of the manuscript. This research was supported by a Whitehall Foundation grant \#20121221 (R.W.), a NSF CRCNS grant \#1308174 (W.L.S.) and \#1308159 (R.W.), and startup funds from the Department of Physics at the University of Arkansas (W.L.S.). We thank J. Gallant for sharing motion-enhanced movie stimuli.

\section{Author contributions}

W.L.S. and R.W. conceived the study and designed the experiments. J.P. designed the visual stimuli. W.L.S., W.P.C., J.P., N.C.W. and R.W. performed the experiments. W.L.S and W.P.C. analysed the data. W.L.S. and Y.K. performed the model simulations. W.L.S. and R.W. wrote the paper.

\section{Additional information}

Supplementary information is available in the online version of the paper. Reprints and permissions information is available online at www.nature.com/reprints. Correspondence and requests for materials should be addressed to W.L.S.

\section{Competing financial interests}

The authors declare no competing financial interests. 


\section{Methods}

Ex vivo eye-attached whole-brain preparation. All procedures were approved by the Institutional Animal Care and Use Committees of both Washington University and University of Arkansas, and conform to the guidelines of the National Institutes of Health on the Care and Use of Laboratory Animals. Adult red-eared turtles ( $n=9$, Trachemys scripta elegans, 150-200 g weight, $12-15 \mathrm{~cm}$ carapace length) were studied. Following anaesthesia (Propofol $10 \mathrm{mg} \mathrm{kg}^{-1}$ ) and decapitation, we surgically removed the brain, optic nerves and eyes, from the cranium (Supplementary Information 9). One eye was hemisected and drained, thus exposing the retina for visual stimulation; the other eye was removed. Two cuts allowed the cortex to be unfolded, exposing the ventricular surface, thus facilitating the subsequent insertion of the microelectrode array. The eye and the brain were continuously perfused with artificial cerebrospinal fluid (in $\mathrm{mM} ; 85$ $\mathrm{NaCl}, 2 \mathrm{KCl}, 2 \mathrm{MgCl}_{2}, 45 \mathrm{Na} \mathrm{HCO}_{3}, 20 \mathrm{D}$ glucose and $3 \mathrm{CaCl}_{2}$, bubbled with $95 \%$ $\mathrm{O}_{2}$ and $5 \% \mathrm{CO}_{2}$ ), adjusted to $\mathrm{pH} 7.4$ at room temperature. Recordings began 2-3 h after induction of anaesthesia.

Microelectrode array measurements. We recorded wideband $(0.7 \mathrm{~Hz}-15 \mathrm{kHz})$ extracellular voltages relative to a silver chloride pellet electrode in the bath at $30 \mathrm{kHz}$ sample rate (Blackrock Microsystems, Cerebus). With post-processing filtering (bandpass $5-100 \mathrm{~Hz}$ ) we extracted the local field potential (LFP). We used two different electrode arrays. The first was a 96 -channel microelectrode array (10 $\times 10$ square grid, $400 \mu \mathrm{m}$ inter-electrode spacing, $500 \mu \mathrm{m}$ electrode length, no corner electrodes, Blackrock Microsystems). Using a micromanipulator (Sutter, MP-285), we inserted this array to a depth of 250-500 $\mu \mathrm{m}$, with the plane of electrodes parallel to the dorsal surface of the cortex. The second array was comprised of a three-dimensional (3D) grid of electrodes $(4 \times 4 \times 8$ grid, 16 shanks, 8 electrodes per shank, $300 \mu \mathrm{m}$ inter shank spacing, $100 \mu \mathrm{m}$ inter-electrode spacing on each shank, Neuronexus). This second array was inserted to a depth such that electrodes spanned the cortex from the ventricular to the dorsal surface. We analysed data from electrodes that were located within the visually responsive region of the cortex (Supplementary Information 2). This included between 13 and 28 electrodes ( 19 on average) for $10 \times 10$ electrodes, and approximately 48 electrodes for the $3 \mathrm{D}$ grid electrodes.

Visual stimuli. Visual stimuli (two types of greyscale movie, black dots moving on a white background, uniform black to grey transition) were created by a computer and delivered with either a miniature video projector (Aaxa Technologies, P4X Pico Projector) or an LCD monitor (Samsung 19", 1,440 $\times 900$ pixels, contrast ratio $=20,000: 1$, response time $=2 \mathrm{~ms}$ ). The projector/monitor image was focused onto the retina with additional lenses (Fig. 1a). The mean light intensity (irradiance) at the retina was $20 \mathrm{~mW} \mathrm{~m}^{-2}$ for the monitor and $1 \mathrm{~W} \mathrm{~m}^{-2}$ for the projector. In two experiments, we also used a brief flash from a light-emitting diode (LED, $60 \mathrm{~W} \mathrm{~m}^{-2}$ at retina) placed near the retina to stimulate with a $1 \mathrm{~s}$ flash. Further details about the stimuli, including timing, are in Supplementary Information 10.

Avalanche analysis. The first step of avalanche detection was to compute the standard deviation of every LFP trace. Next we defined an 'LFP peak' as a period of time during which an LFP trace fluctuates beyond 3 to 4 standard deviations, owing to either a positive or negative deflection (Fig. 1b). For each LFP peak, we determined the time of its extreme value and the identity of the channel on which it was recorded. The channel information was used to exclude from analysis LFP peaks which were not within visual cortex. An avalanche was defined as a spatiotemporal cluster of consecutive LFP peaks with inter-peak intervals not exceeding a temporal threshold $\Delta T$ (channel information does not play a role in avalanche definition). $\Delta T$ was chosen to be the average inter-peak interval (〈IPI , inverse of population LFP peak rate), resulting in $\Delta T=24 \pm 18 \mathrm{~ms}$ (mean \pm s.d.). Avalanche duration was defined as the difference between the first and last LFP peak time within the avalanche. The size of an avalanche was defined as the number of LFP peaks comprising the avalanche. Avalanches were analysed separately depending on whether they occurred during the transient period or visually driven steady state period. Robustness of model and experimental results to changes in $\Delta T$ and definitions of time periods are described in Supplementary Information 11 .

Power-law fitting and fit quality, $q$. Using maximum likelihood methods ${ }^{10,18}$, we fit a truncated power law (truncated at both the head and tail) to the avalanche distributions during visually driven steady state (Supplementary Information 12). The fitting function for the avalanche size distribution was

$f(S)=S^{-\tau}\left(\sum_{x=x_{0}}^{x_{M}} x^{-\tau}\right)^{-1}$, where the maximum size $x_{M}$ was assumed to be the largest observed size. The minimum size $x_{0}$ and the exponent $\tau$ were fitting parameters. As avalanche duration is a non-integer variable, the fitting function for the duration distribution was $g(D)=(1-\alpha)\left(y_{M}^{1-\alpha}-y_{0}^{1-\alpha}\right)^{-1} D^{-\alpha}$, where the maximum $y_{M}$ was taken as the largest observed duration, and $y_{0}$ and $\alpha$ were fitting parameters. Exponents $\tau$ and $\alpha$ between 1 and 4 in increments of 0.01 were tried. Minimum values $x_{0}$ and $y_{0}$ were tried, increasing from 0 , but only up to the point when the fitted power law matches the data well enough to have a

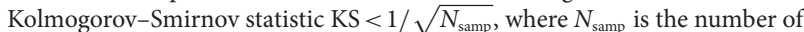
avalanches comprising the data set (Supplementary Information 12). For fitting model data size and duration distributions, we used the fitting function $f(S)$ above, because both size and duration are discrete variables for the model.

After finding the best-fit power law, the next step was to assess goodness-of-fit $q$ (refs 10,18$)$. We compared the experimental data against 1,000 surrogate data sets drawn from the best-fit power-law distribution with the same number of samples as the experimental data set. The deviation between the surrogate data sets and a perfect power law was quantified with the KS statistic. The quality $q$ of the power-law fit was defined as the fraction of these surrogate KS statistics which were greater than the KS statistic for the experimental data. We use a very conservative criterion, $q>0.1$, for judging the data to be power-law distributed. This is demonstrated visually in Fig. 1f,g and Fig. 2e,f by plotting the experimental distribution over a grey band which delineates the 5th-95th percentiles of the surrogate data sets.

Computational model. A total of $N=1,000$ all-to-all connected binary neurons received input from outside the network. The 'strength' of the synapse from neuron $j$ onto neuron $i$ at time $t$ is determined by the corresponding element of the synaptic weight matrix $W_{i j}(t) .20 \%$ of neurons are inhibitory-that is, with negative entries in the weight matrix. $\Omega_{i}(t)$ is the strength of the input synapse onto neuron $i$ (all excitatory). The binary state $s_{i}(t+1)$ of neuron $i$ ( $s=0$ inactive, $s=1$ spiking $)$ is determined probabilistically based on the sum $p(t+1)$ of its inputs $p(t+1)=\Omega_{i}(t) \sigma_{i}(t)+\sum_{j=1}^{N} W_{i j}(t) s_{j}(t)$. If $0<p<1$, then the neuron fires with probability $p$. If $p \geq 1$, then the neuron fires with probability 1 . If $p \leq 0$, then the neuron does not fire. Time is discrete and state updates are synchronous. The input $\sigma_{i}(t)$ from the $i$ th input synapse is binary (1 with probability $r$ ). The onset of stimulation is modelled as a step increase from $r=5 \times 10^{-5}$ to either $r=0.02$ or $r=0.1$. In Figs 2 and 3, we report the population input rate of $R=N r$. The update rules for synaptic dynamics are $W_{i j}(t+1)=W_{i j}(t)+\tau_{r}{ }^{-1}\left(W_{i j}^{o}-W_{i j}(t)\right)-\tau_{d}{ }^{-1}$ $W_{i j}(t) s_{j}(t)$ and $\Omega_{i}(t+1)=\Omega_{i}(t)+\tau_{r}{ }^{-1}\left(\Omega_{i}^{o}-\Omega_{i}(t)\right)-\tau_{d}{ }^{-1} \Omega_{i}(t) \sigma_{i}(t)$. The default weight matrix was constructed such that its largest eigenvalue $\Lambda_{0}$ has absolute value equal to either 1.0, 1.05, or 1.1 (Supplementary Information 7). A largest eigenvalue of 1.0 corresponds approximately to an average synaptic weight of $1 / \mathrm{N}$ and is known to result in critical dynamics for models with static synapses ${ }^{26}$. Synapses depress with a time constant of $\tau_{d}=20$ timesteps following a presynaptic spike, then recover exponentially with a time constant of $\tau_{r}=400$ timesteps.

Each avalanche is initiated by external input. On reaching a timestep with no active cortical neurons, the avalanche is considered to be ended. We simulated 40 trials of step increase in input. In each trial, we ran the model for 5,000 timesteps following the onset of increased input. As in previous studies ${ }^{25,27,33}$, subsampling (Fig. 3) entailed analysing the spikes from a randomly chosen $30 \%$ or $10 \%$ of the network. More extreme subsampling, which matches experimental LFP peak rate with model spike rate, are explored in Supplementary Information 7.

\section{References}

33. Priesemann, V., Munk, M. H. J. \& Wibral, M. Subsampling effects in neuronal avalanche distributions recorded in vivo. BMC Neurosci. 10, 40 (2009). 